\title{
Something for everyone
}

\section{Horizontal gene transfer in evolution • by Charles G. Kurland}

Horizontal gene transfer (HGT) is a sensational topic largely because its exploitation to date has been primarily journalistic. Properly packaged it captures the attention of a sensitized lay public and peps up circulation. HGT provides technical challenges for academic scientists as well as market analysts. For fans of science fiction as well as for environmental pessimists, HGT is the stuff of doomsday prophecies. Interest in HGT might even be seen as a reasonable response to the recent acquisition of genome sequence data and the need to understand how genomes exchange sequences.

For fans of science fiction as well as for environmental

pessimists, HGT is the stuff of doomsday prophecies

There is an unaccountable frenzy in the academic literature surrounding horizontal gene transfer. For example, we read that the discovery of HGT in recently sequenced genomes has suggested a new paradigm and that we can 'let go' of phylogenetic classification. We may even 'define organisms as more than the sums of their genes and imagine organismal lineages to have some sort of emergent reality' (Doolittle, 1999). We learn that 'much of the speciation and subspeciation in bacteria can be explained as the result of macro-evolutionary events mediated by HGT' (de la Cruz and Davies, 2000). Likewise, we are told that bacteria adapt to new environments by HGT and not by single nucleotide substitutions (Ochman et al., 2000). Earlier, we were told that operon structures in bacteria are the creations of HGT (Lawrence and Roth, 1996). All of these claims are part of what I call global HGT, an ideology that is begging for deconstruction.

It is certainly true that instances of HGT were discovered in the first genome sequences. Quite reasonably, such discoveries fired up criticism of the notion that any randomly chosen gene family can be used to establish the phylogenetic status of an organism. In particular, the reigning protocol for phylogenetic inference, which employs the gene family of ribosomal RNA (rRNA) was challenged. Woese (1987) had earlier described in detail the ubiquity, conservative features as well as other virtues that recommend this unique gene family as a universal reference for sytematics as well as for phylogeny. In his critique of this 'paradigm' Doolittle (1999) emphasizes the potential uncertainties introduced by HGT to phylogeny based on rRNA and presents examples of apparently incorrect phylogenetic inferences from rRNA sequences. But it needs to be said that not a single instance of phylogenetic ambiguity resulting from the horizontal transfer of rRNA genes has been described. The failure to find such an example of ambiguous rRNA phylogeny was not from want of trying.

HGT generates phylogenetic networks among phylogenetic trees; that is to say, HGT is expressed as reticulate (network) evolution. Reticulation is certainly a complication but it is not the bête noire of phylogeny. Thus, HGT only marginally increases the complexity of the bookkeeping; it does not destroy the possibility of keeping track of evolution. For example, median joining networks (MJN) have been used for intraspecific phylogenetic reconstructions that incorporate reticulation in a transparent way (Bandelt et al., 1999; Kivisild et al., 1999). Hopefully, the next generation of MJN algorithms will accommodate HGT in the reconstruction of interspecific phylogeny.
Like HGT, sequence duplications, deletions and inversions mark singularities in the evolution of a genome. These are certainly more cumbersome than single nucleotide substitutions but they do not obviate phylogeny. They merely introduce 'gaps' in genic or in genomic alignments that slightly complicate phylogenetic reconstruction.

Sequence comparisons that exploit orthologues present exclusively in one or another phylogenetic cluster provide ways to track genomic lineages even when HGT is rampant. Thus, Woese (1998) has suggested that HGT is not a random process that transfers any coding sequence across phylogenetic boundaries. Instead, HGT will be selective for donor sequences that are compatible with the genomic make up of the acceptor lineage. Cooperative interactions within functional protein complexes might not accommodate the donor gene product. Consequently, gene products that function more or less solo will have the greatest chance to be transferred to distantly related cells. Thus, HGT for aminoacyltRNA synthetases is observed, and it is found that the synthetases are exchanged in both directions between prokaryotes and eukaryotes (Woese et al., 2000).

Not a single instance of phylogenetic ambiguity resulting from the horizontal transfer of rRNA genes has been described

Occasionally, a transferred gene product may actually find itself in a particularly friendly molecular environment in its new host. If this occurs, selection may fix the alien gene in its adoptive lineage making it a useful phylogenetic marker for all 


\section{viewpoint}

of the descendants of the lineage that propagate the interloper. It is already possible to identify hundreds of orthologues that are specific to archaea (Graham et al., 2000) or to eukaryotes (O. Karlberg, B. Canbaeck, C.G. Kurland and S.G.E. Andersson, submitted), and more are to be expected. These taxa-specific orthologs define the genomic signature of phylogenetic clusters and can be used in more sophisticated ways to establish detailed, robust phylogenetic reconstructions even when HGT is rampant (Graham et al., 2000).

As noted above, extravagant claims have been made for the pervasive influence of HGT on the origin of species. Of course, the efficiency with which transferred genes may be established in bacterial lineages under special circumstances is impressive. One need only consider the spread of plasmid-borne multiple-antibiotic resistance among bacterial pathogens to realize how successful this mode of adaptation is when sudden selective pressures are imposed. Similarly, there are well documented adaptive functions for plasmid-borne gene products as diverse as those that degrade aromatics and other organic molecules, to ones that enhance pathogenic virulence, all of which can cross phylogenetic boundaries (de la Cruz and Davies, 2000; Ochman et al., 2000). These functions may be supplemented by additional modes of HGT such as transposon transfer, viral transduction and DNA transformation. Little wonder then that there are numerous examples of HGT among the newly sequenced genomes of archaea and bacteria.

Are we to believe that most of the novel adaptive evolution of prokaryotes is dominated by $6 \%$ of alien genes?

Ochman et al. (2000) have summarized sightings of transferred genes in 19 prokaryotic genomes. They found that the detectable fraction of alien coding sequences varies from nearly zero in some endocellular parasites to $\sim 17 \%$ in Synechocystis. I reckon that the mean value for the incidence of alien genes in these 19 genomes is a bit more than $6 \%$. Now, are we to believe that most of the novel adaptive evolution of these prokaryotes is dominated by this $6 \%$ ? Alternatively, have the other $94 \%$ of these genomes simply been passive hitchhikers when HGT boldly takes bacteria to places no bacterium has gone before? I doubt it.

In the first place, environmental changes are usually not as rapid as the sudden and wanton use of antibiotics has been in recent decades. For example, we might expect the adaptive response of

rearrangements including the duplication of an entire genome sequence provide other modes of expansive evolution (Wolfe and Shields, 1997). Zuckerkandl and Pauling (1965) inferred that gene duplications followed by evolutionary diversification would provide a mechanism to enrich the genetic repertoire of organisms. This is massively verified by the detection of large paralogous gene families in all genomes studied to date.

Diversification of paralogous genes can also generate proteins that act on different steps of a single biochemical pathway (Zuckerkandl and Pauling, 1965). Furthermore, intraspecific recombination can drive the genes for interactive proteins into neighbouring genomic configurations that might facilitate their co-evolution and regulation (Lawrence and Roth, 1996). Of course, such constellations may be transferred by HGT to distantly related organisms. But this would require strong selective pressures as in the case of multiple antibiotic resistance because long sequences provide large targets for mutational inactivation. For multigenic strings that are only weakly or sporadically selective, the takeover of a population is not a likely event. It is more likely that the string will be mutation-

microbes to geological or climatic changes to be commensurately gradual. Similarly, the progression of genomic changes from a free-living bacterium to an endocellular symbiont or to a parasite is not sudden. Indeed, it is possible to observe the recent signs of systematic evolutionary change in the genomes of bacteria that began to adapt to the intracellular milieu many millions of years ago (Andersson and Kurland, 1998a; Andersson and Andersson, 1999).

Ochman et al. (2000) have emphasized that there are greater creative possibilities resulting from HGT than those attending single nucleotide substitutions. This is a preposterously narrow comparison. It is well known that single nucleotide substitutions are not the only mode of evolution by intraspecific mechanisms. Genomic ally inactivated before it can reach fixation (Andersson and Kurland, 1998b). Indeed, there is significant variability observed for the genomic arrangements of sporadically selected multigenic arrays (Lawrence and Roth, 1996), which most likely reflects the inroads of random mutation (Andersson and Kurland, 1998b).

The impact of HGT on the adaptation of bacteria to new environments is limited by the vagaries of selection. Mercifully, only a small fraction of pathogenic bacteria in the world carry antibiotic-resistance plasmids. The limited distribution of these plasmids within a bacterial population probably reflects the fact that only some habitats of bacteria are selective for antibiotic resistance whereas in others the resistance plasmids may be lost or never 


\section{viewpoint}

selected. It seems to me that mobile elements that circulate in bacterial populations such as plasmids are less attractive as vehicles for adaptive speciation than they are valuable indicators of the genomic diversity of bacterial species. These elements are either periodically selected or vanish by mutational attrition. Nevertheless, when an intact mobile element is still circulating in a bacterial population, it is a potential genomic recruit that can facilitate the adaptation of its hosts to one or another environmental challenge.

The notion that HGT can play the dominant role in speciation (de la Cruz and Davies, 2000) is probably a misunderstanding. I hesitate to wade into the swamp that is bacterial speciation. However, it seems to me that speciation must be a phenomenon dominated by vertical evolution because under conditions in which HGT dominates over vertical evolution the notion of a species loses all meaning. So, for example, in his discussions of the properties of the ancestral progenote, Woese (1998) has emphasized the idea that pervasive HGT allows rapid testing of novel genetic combinations in primitive biological systems. However, as soon as the combinatorial interactions approach some sort of optimality, exclusive boundaries are established on a molecular level and eventually on a cellular level. That is to say there is a transition from primitive HGT-dominated biology to the vertical gene transmission we are most familiar with.

HGT can certainly confer adaptive properties that contribute to speciation especially under conditions of rapid environmental change. However, I suspect that much else is needed in addition to transform the host of newly acquired mobile elements into a new species. For example, it might be necessary for the organism to become restricted to the selective environment and to be prevented from cycling through non-selective environments for long periods of time. One way for this to happen would be for mutations to inactivate the genetic determinants that support the host in environments other than those that are selective for the mobile elements. This could hardly be a rapid process and it would almost certainly require extensive genomic evolution.

Sequence comparisons of homologues have hinted at extensive HGT from bacteria to eukaryotes (Doolittle et al., 1996; Feng et al., 1997; Rivera et al., 1998). However, interpretations of these particular data are difficult because they consist for the most part of two taxon comparisons that relate sequence similarities only. The multiple taxon comparisons that would yield phylogenetic trees are still not available. Consequently, pinpointing the phylogenetic origins of these putative gene transfers is not possible. This did not deter Gogarten et al. (1996) from suggesting that the putative bacterial descendants observed in eukaryotes are ancient souvenirs of the endosymbiotic bacteria that seeded the emergent eukaryotic cell with mitochondria or plastids. Absolutely nothing indicates that these putative transferred genes are the descendants of $\alpha$-proteobacteria or cyanobacteria. Furthermore, Jain et al. (1999) suggest that the transferred genes did not appear in eukaryotic lineages in a single burst but have been transferred intermittently over time.

Such gradual transfer is consistent with current notions of how prokaryotic genes might be transferred to eukaryotic genomes. Briefly, archaea or bacteria may be digested by the phagolysosomes of eukaryotic cells, as are mitochondria (Thorsness and Fox, 1990; Hanekamp and Thorsness, 1996). Release of nucleic acid fragments from the phagolysosomes is thought to provide opportunities for transformation of the nuclear genome. In this way the neutral diffusion of coding sequences from mitochondria or bacterial guests may transfer genes into the eukaryotic nucleus (Berg and Kurland, 2000). However, there is a catch. In order to be propagated in the descendants of an organism, the transfer must be made within the germ line of the new host. This creates no problem for HGT in unicellular creatures, but in higher eukaryotes there may be barriers. Some higher eukaryotes such as arthropods maintain endosymbiotic bacteria that are inherited vertically via eggs (Stouthamer et al., 1999). But if you ask your family doctor how often she has come across a primary bacterial infection of the ovaries or the testes, she will almost certainly answer 'never'. Similarly, some plants enjoy the benefits of bacterial plasmid-borne functions such as nitrogen fixation in the associations of legumes with Rhizobium (Freiberg et al., 1997). There may be strong selective pressures to incorporate nitrogen fixation genes into the nuclear genome of legumes but these sequences have never gained access to the germ line. In brief, the genomes of many of the higher eukaryotes may not be accessible to HGT from bacteria. However, HGT associated with retroviruses, DNA transposons and retrotransposons is rife among the higher eukaryotes. It inserts characteristic repeat sequences in eukaryotic genomes that may make up a substantial fraction of a genome.

\section{Unconstrained use of hypothetical HGT generates all sorts of paper scenarios}

Finally, nothing illustrates the global HGT circus as well as the plethora of models for the origin of the eukaryotic cell. Following hints that some eukaryotic genes are more closely related to archaeal homologues while others may descend from bacterial homologues has been a fruitful way to generate models for the origin of eukaryotic cells. These include scenarios with cell fusion, engulfment or symbiosis (Lopez-Garcia and Moreira, 1999). The problem of course is that unconstrained use of hypothetical HGT generates all sorts of paper scenarios. Obtaining phylogenetic data with which to distinguish the alternatives is not as easy.

Armed with the strategy of downsizing the national infrastructures while demanding that science instantly enriches nations, the global market has managed at last to force the gates to the ivory tower. Scientists now perceive that their survival depends on being heard above the clamour of the global marketplace. So, just as happened in the public media long ago, balanced science reporting is now being abandoned for sensation and self-advertising. Nothing in science is more self-aggrandizing than the claim that 'all that went before me is wrong', except perhaps the claim that 'I can explain everything'. Proponents of global HGT have it both ways.

I am very grateful to Tomoko Ohta, Richard Vilhems and Carl Woese for helpful discussions and criticism.

Andersson, J.O. and Andersson, S.G.E. (1999) Insights into the evolutionary process of genome degradation. Curr. Opin. Genet. Dev., 9, 664-671. 


\section{viewpoint}

Andersson, S.G.E. and Kurland, C.G. (1998a) Reductive evolution of resident genomes Trends Microbiol., 6, 263-268.

Andersson, S.G.E. and Kurland, C.G. (1998b) Ancient and recent horizontal transfer events: the origins of mitochondria. APMIS Suppl., 84, 5-14.

Bandelt, H.-J., Forster, P. and Rohl, A. (1999) Median-joining networks for inferring intraspecific phylogenies. Mol. Biol. Evol., 16, $37-48$.

Berg, O.G. and Kurland, C.G. (2000) Why mitochondrial genes are most often found in nuclei. Mol. Biol. Evol., 17, 951-961.

Campbell, C.L. and Thorsness, P.E. (1998) Escape of mitochondrial DNA to the nucleus in yme1 yeast is mediated by vacuolar-dependent turnover of abnormal mitochondrial compartments. J. Cell Sci., 111, 2455-2464.

de la Cruz, F. and Davies, J. (2000) Horizontal gene transfer and the origin of species: lessons from bacteria. Trends Microbiol., 8, 128-133.

Doolittle, R.F., Feng, D.-F., Tsang, S., Cho, G. and Little, E. (1996) Determining divergence times of the major kingdoms of living organisms with a protein clock. Science, 271, 470-477.

Doolittle, W.F. (1999) Phylogenetic classification and the universal tree. Science, 284, 2124-2129.

Feng, D.-F., Cho, G. and Doolittle, R.F. (1997) Determining divergence times with a protein clock: update and reevaluation. Proc. Natl Acad. Sci. USA, 94, 13028-13033.

Freiberg, C., Fellay, R., Bairoch, A., Broughton, W.J., Rosenthal, A. and Perret, X. (1997) Molecular basis of symbiosis between Rhizobium and legumes. Nature, 387, 394-401.

Gogarten, J.P., Olendzenski, L., Hilario, E. Simon, C. and Holsinger, K.E. (1996) Dating the cenancester of organisms. Science, 274, 1750-1751.

Graham, D.E., Overbeek, R., Olsen, G.J. and Woese, C.R. (2000) An archaeal genomic signature. Proc. Natl Acad. Sci. USA, 97, 3304-3308.

Hanekamp, T. and Thorsness, P.E. (1996) Inactivation of YME2, an integral inner mitochondrial membrane protein, causes increased escape of DNA from mitochondria to the nucleus in Saccharomyces cerevisiae. Mol. Biol. Cell, 16, 2764-2771.

Jain, R., Rivera, M.C. and Lake, J.A. (1999) Horizontal gene transfer among genomes: the complexity hypothesis. Proc. Natl Acad. Sci. USA, 96, 3801-3806.

Kivisild, T. et al. (1999) Deep common ancestry of Indian and western-Eurasian mitochondrial DNA lineages. Curr. Biol., 9, 1331-1334.

Lawrence, J.G. and Roth, J.R. (1996) Selfish operons: horizontal trnasfer may drive the evolution of gene clusters. Genetics, 143, 1843-1860.

Lopez-Garcia, P. and Moreira, D. (1999) Metabolic symbiosis at the origin of eukaryotes. Trends Biotechnol., 24, 88-93.

Ochman, H., Lawrence, J.G. and Groisman, E.A (2000) Lateral gene transfer and the nature of bacterial innovation. Nature, 405, 299-304.

Rivera, M.C., Jain, R., Moore, J.E. and Lake, J.A (1998) Genomic evidence for two functionally distinct gene classes. Proc. Natl Acad. Sci. USA, 95, 6239-6244.

Saier, M.H. and Paulsen, I.T. (1999) Paralogous genes encoding transport proteins in microbial genomes. Res. Microbiol., 150, 689-699.

Stouthamer, R.J., Breeuwer, A.J. and Hurst, G.D.D. (1999) Wolbachia pipientis: microbial manipulator of arthropod reproduction. Annu. Rev. Microbiol., 53, 71-102.
Thorsness, P.E. and Fox, T.D. (1990) Escape of DNA from mitochondria to nucleus in Saccharomyces cerevisiae. Nature, 346, 376379 .

Woese, C.R. (1987) Bacterial evolution Microbiol. Rev., 51, 221-271.

Woese, C.R. (1998) The universal ancestor. Proc. Natl Acad. Sci. USA, 95, 6854-6859.

Woese, C.R., Olsen, G.J., Ibba, M. and Soell, D. (2000) Aminoacyl-tRNA synthetases, the genetic code, and the evolutionary process. Microbiol. Mol. Biol., 64, 202-236.

Wolfe, K.H. and Shields, D.C. (1997) Molecular evidence for an ancient duplication of the entire yeast genome. Nature, 387, 708-713.

Zuckerkandl, E. and Pauling, L. (1965) Molecules as documents of evolutionary history. J. Theor. Biol., 8, 357-366.

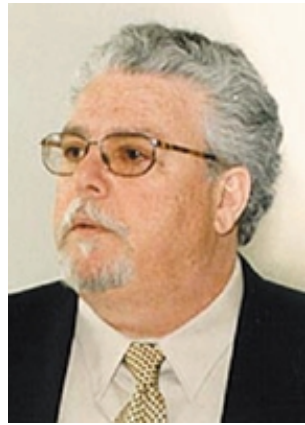

The author is at the Department of Molecular Evolution, Evolutionary Biology Centre, Uppsala University, Uppsala and the Department of Microbiology, University of Lund, Lund, Sweden.

DOI: 10.1093/embo-reports/kvd042 